\title{
The impact of anecdotal information on medical decision-making
}

\author{
Sara Jaramillo (sdjarami@asu.edu) \\ School of Social and Behavioral Sciences, Arizona State University \\ Phoenix, AZ, USA \\ Zachary Horne (Zachary.Horne@asu.edu) \\ School of Social and Behavioral Sciences, Arizona State University \\ Phoenix, AZ, USA \\ Micah Goldwater (micah.goldwater@sydney.edu.au) \\ School of Psychology, The University of Sydney \\ Camperdown, Australia
}

\begin{abstract}
Prior research has found that arguments that use both anecdotal and statistical evidence are more persuasive than arguments using either alone (Allen, Bruflat, Fucilla, Kramer, McKellips, Ryan, \& Spiegelhoff, 2000; Hornikx, 2005). However, it isn't clear how people integrate anecdotal and statistical information when making medical decisions, particularly when this information is in conflict. In three preregistered experiments, we tested how people integrate conflicting information to judge the efficacy of a new medical treatment. Participants read either an anecdote from someone in a clinical trial, summary statistics about the clinical trial, or both types of information. We found that reading an anecdote about a participant in the clinical trial for whom the treatment was ineffective reduced people's beliefs in the medical treatment, even when participants received strong evidence that the treatment was effective. We also found that visually presenting statistical information increased the perceived efficacy of the treatment but did not eliminate the effect of the anecdote.
\end{abstract}

Keywords: anecdotal reasoning; medical decision-making; open science

\section{Introduction}

Making decisions about medical treatments can be a difficult and stress inducing process. When the decision concerns those we love, or those who are vulnerable, the stakes can make even obvious decisions seem paralyzing. People are inundated with popular press reports about medical research concerning what's healthy, get advice from doctors, and hear personal anecdotes from friends, relatives, and the media. How can people make appropriate medical decisions under these conditions? It might seem obvious that people's beliefs should reflect the scientific consensus, but when our own and our families' health is at stake, a compelling narrative or personal anecdote can be hard to ignore. For instance, vaccine hesitancy has been found to be driven by reliance on anecdotal evidence about the side effects of vaccines spread throughout online communities even though vaccines are among the safest medical treatments (Powell, Weisman, \& Markman, 2018). Altogether making a medical decision is no easy feat, even for the epistemically diligent.

Prior research suggests that although people are capable of correctly integrating statistical information to make informed medical decisions (e.g., Allen \& Preiss, 1997;
Allen et al., 2000; Hornikx, 2005), they may nonetheless improperly attend to irrelevant anecdotal information, particularly when that evidence is salient and relates to uncertainty and risk (e.g., Allen et al., 2000; Shen, Sheer, \& $\mathrm{Li}, 2015)$. Some researchers suggest that narratives are more engaging and comprehensive (Dahlstrom, 2014), but when learning about new scientific information, anecdotal information can distract from making proper scientific judgments (Rodriguez, Rhodes, Miller, \& Shah, 2016). What remains unclear is how people integrate anecdotes with statistical information. When people are presented with both statistical summary information and anecdotes, how do they reason on the basis of this information? Can positive anecdotal information aid in the integration of statistical information when in concert with each other? Some research suggests that anecdotes do not impact the integration of statistical evidence about government policy (Hornikx 2018), but there is little research on this question in the domain of medical decision-making, where the stakes are high and thus anecdotes might may exhibit stronger effects.

In the present studies, we examined the effect of anecdotes on medical decision-making. We investigated the ways in which anecdotal information influences how people interpret a study describing the efficacy of a novel medical treatment (Experiments 1 and 2), and what other factors may weaken the effect of anecdotes on reasoning (Experiment 3 ).

\section{General Methods}

Preregistration We preregistered the data collection plan, analyses, and predictions for all three experiments. Experimental scripts, full analytic results, and supplementary online materials (SOM) are available on the Open Science Framework at https://osf.io/dkcwv/.

Analytic Approach We performed Bayesian estimation using the $\mathrm{R}$ package brms (Bürkner, 2018). We set regularizing priors for all population-level effects in our models, which we detail below. These priors are recommended because they provide conservative effect size estimates and reduce the likelihood of overfitting (McElreath, 2016). Following the recommendations of 
Liddell \& Kruschke (2018), Likert data were modeled with a cumulative probability distribution.

\section{Experiment 1}

Experiment 1 examined how anecdotes affect people's reasoning about medical information. We sought to avoid polarizing medical treatments because beliefs about these topics may be particularly intransigent. Consequently, we focused on a plausible but relatively unknown medical treatment that people would not have strong beliefs about. Specifically, we examined people's beliefs about B-12 injections as means for treating chronic headaches.

Participants We recruited 497 participants through Amazon's Mechanical Turk (47\% women, $M_{\text {age }}=38$ years old). Participants were paid $\$ 0.50$ for participating in a five-minute study. After excluding participants who missed questions checking their attention, 431 participants remained in our sample. Our exclusion criteria were determined a priori and were in accordance with our study preregistration.

Procedure In Experiment 1, we presented participants with either statistical evidence, anecdotal evidence, or the combination of both types of evidence about a medical trial testing the effectiveness of B-12 injections on chronic headaches. The study consisted of three parts: a pretest questionnaire, an intervention, and then a posttest questionnaire. After completing this portion of the study, participants completed medical individual differences measures and demographic questions. We describe each component below.

Pretest Questionnaire Participants answered a brief questionnaire examining their familiarity with B-12 injections, whether they are currently receiving or have received B-12 injections, and whether they are considering receiving B-12 injections as a medical treatment. After responding to these questions, participants were then asked on a five-point Likert scale whether they believe B-12 injections are an effective medical treatment $(1=$ "Not effective at all", 5 = "Extremely effective").

Conditions After completing the B-12 pretest questionnaire, participants were randomly assigned to one of four conditions: the Statistics condition, the Positive Anecdote condition, the Statistics + Positive Anecdote condition, or the Statistics + Negative Anecdote condition.

In the Statistics condition, participants were shown a description with summary statistics about a clinical trial examining the effects of B-12 injections on patients with chronic headaches. Namely, participants read that in a clinical trial with 1,000 subjects, B-12 injections were $87.3 \%$ effective as a medical treatment for chronic headaches.

In the Positive Anecdote condition participants did not receive the statistical information but were told "Jamie's [the protagonist in the anecdote] doctor recommended that she participate in a new clinical trial that was examining the effects of B-12 on headaches" and then were told that Jamie decided to receive B-12 and subsequently experienced a reduction in her symptoms.

In the Statistics + Positive Anecdote condition, participants first read the summary statistics demonstrating the efficacy of B-12 injections (that is, the only material presented in the Statistics condition). They were then told that they would read about the experience of one of the subjects in the study, after which they were presented with the anecdote from the Positive Anecdote condition.

Participants in the Statistics + Negative Anecdote condition were given the same materials as participants in the Statistics + Positive Anecdote condition, but now the anecdote is from a member of the trial for whom treatment was ineffective. Participants learned that "Jamie received a B-12 injection and her headaches, lack of energy, and inability to focus persisted." Critically, however, Jamie was not described as experiencing any side-effects as a consequence of her treatment.

Two design decisions are important to highlight: First, the anecdote contains no new information in the conditions that paired an anecdote with a statistic. This is because summary statistics already capture the success or failure of B-12 injections in the clinical trial and the anecdote concerns someone who was in the clinical trial. In other words, the anecdote contains no additional information over and above the statistic - the anecdote either describes the treatment as effective or ineffective and no other relevant information beyond this.

This point is related to a second design decision: Namely, in the Negative Anecdote condition, B-12 was described as failing as a treatment but not introducing any unwanted side-effects. Together, then, the negative anecdote should not affect participants' interpretation of the statistical information presented to them.

Posttest Questionnaire After completing the intervention portion of the task, participants completed a posttest questionnaire in which they were asked whether they believed B-12 injections were an effective medical treatment.

As noted, one possibility is that when the stakes are high for a given medical decision, people may be more susceptible to anecdotal information leading them to ignore strong statistical information. To this end, we also included two additional questions in the posttest questionnaire. First, participants were asked how likely it was they would try B-12 injections on a five-point Likert scale. Second, they were asked how likely they were to give B-12 injections to their child (if applicable). It's possible that a negative anecdote would exhibit a stronger negative effect on people's reasoning about their child compared to themselves because people are more risk averse when it comes to making decisions that impact their children's health (e.g., Brody, Annett, Scherer, Perryman, \& Cofrin, 2005; Johnson, Özdemir, Mansfield, Hass, Siegel, \& Sands, 2009). 


\section{Predictions}

We predicted that participants in the Statistics + Positive Anecdote condition would be most likely to think that B-12 injections were effective as a treatment for chronic headaches-the positive anecdote would make salient the statistical summary information. This outcome would suggest that health communication experts could include similar positive anecdotes to increase people's uptake of statistical information (Allen et al., 2000). In contrast, we were unsure whether the Statistics condition or the Positive Anecdote condition would differ from each other, though the Statistics condition objectively contains much stronger evidence.

Of particular interest was how participants would respond to the negative anecdote in the Statistics + Negative Anecdote condition. One possibility is that presenting participants with a negative anecdote could raise the salience of the inefficacy of B-12 injections. However, we were unsure to what extent a single negative anecdote could impact people's use of the statistical summary information.

\section{Results}

We tested our predictions by fitting a Bayesian multivariate ordinal regression model regressing B-12 beliefs (i.e., efficacy beliefs, willingness to try B-12, and willingness to give these injections to their children) on Condition (Reference $=$ Positive Anecdote condition) and pretest beliefs about the efficacy of B-12. Following the recommendations of Bürkner and Charpentier (2018), we modeled pretest as a monotonic effect because the ordinal nature of this predictor. The model is specified below in brms syntax:

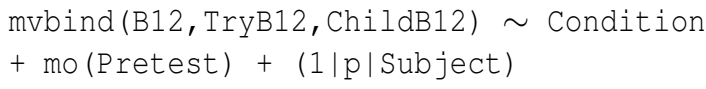

Bayesian analyses formulate model parameters as probability distributions wherein the posterior distribution for a parameter $\theta$ is computed via the prior and the likelihood of $\theta$. To model the joint probability distribution of participants' responses, we specified the following regularizing priors over the possible effects each parameter could have on the response variable:

Experiment 1 - Priors

$$
\begin{aligned}
& \beta_{\text {Intercept }[1]} \sim \mathcal{N}(.5, .5) \\
& \beta_{\text {Intercept }[2]} \sim \mathcal{N}(1.09, .5) \\
& \beta_{\text {Intercept }[3]} \sim \mathcal{N}(2.94, .5) \\
& \beta_{\text {Intercept }[4]} \sim \mathcal{N}(4.59, .5) \\
& \beta_{\text {Pretest }} \sim \mathcal{N}(2,4)
\end{aligned}
$$

All remaining $\beta$ were distributed as $\mathcal{N}(0,1)$

$\Omega_{k} \sim L K J(1)$ where $\Omega_{k}$ is a correlation matrix of group-level parameters

Group-level parameters were distributed as $t(3,0,10)$

This model revealed that the Positive Anecdote, Statistics, and Statistics + Positive Anecdote conditions did not
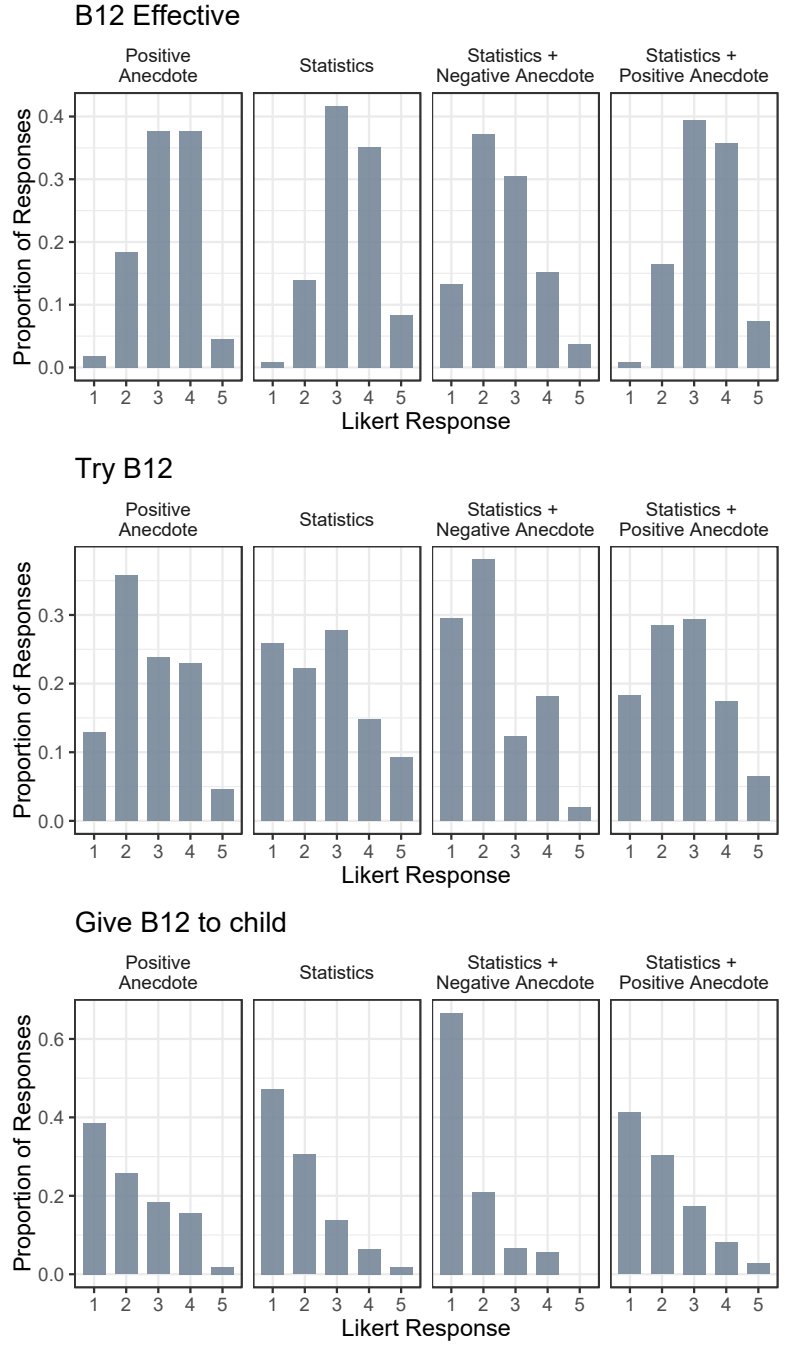

Figure 1: B-12 injection beliefs across conditions in Experiment 1. Higher Likert scale choices indicate more favorable attitudes towards B12 injections. The figure indicates that participants in the Statistics + Negative Anecdote condition had less favorable attitudes towards B12 injections after the intervention relative to participants in the other three conditions.

materially differ from each other (see Figure 1). However, the negative anecdote in the Statistics + Negative Anecdote condition caused participants to ignore the statistical information, despite the fact that (1) the statistic already summarizes the information contained in the negative anecdote and (2) the negative anecdote in no way suggests that the protagonist suffered a side-effect as a result of taking B-12 injections, $b_{B 12}=-1.28,95 \%$ CI $[-1.83,-0.73] ; b_{T r y}=$ $-0.79,95 \%$ CI $[-1.44,-0.15] ; b_{\text {Child }}=-1.39,95 \%$ CI $[-2.07,-0.72]$. A subsequent analysis interacting pretest beliefs with condition provided no evidence for an interaction between these predictors.

Altogether, these findings suggest that (negative) 
anecdotal information affected participants' beliefs. A single positive anecdote carried the same evidential weight as a study describing a double-blind clinical trial with 1,000 participants, though it appears that it did not affect beliefs additively - the Statistics + Positive Anecdote condition did not differ from the Positive Anecdote condition nor the Statistics condition. More worrisome was the effect of the negative anecdote on participants' reasoning about compelling statistical evidence. One negative anecdote, in effect, caused people to dismiss strong statistical evidence, even though the anecdote implied no negative side effects and contained no additional information over and above the information carried by the statistics.

\section{Experiment 2}

Experiment 1 suggested that people's beliefs about the efficacy of B-12 injections are affected by anecdotal information. In Experiment 2, we sought to further understand the impact of anecdotes on medical decision-making. Given that a single negative anecdote can undo, as it were, strong statistical evidence, we sought to determine what would reduce the impact of this negative anecdote. Consequently, we tested whether presenting participants with both a positive and negative anecdote paired with statistical information would lead participants to primarily attend to the statistical information about the efficacy of B-12 injections in treating chronic headaches. Reading contradictory anecdotal information should indicate to participants that a different evidence source (in this case, the statistics) is needed to come to an informed belief about B-12 injections.

\section{Method}

\section{Participants}

We recruited 492 participants through Amazon's Mechanical Turk (50\% women, $M_{\text {age }}=36$ years old). Participants were paid $\$ 0.50$ for participating in the study. After excluding participants who missed questions checking their attention, 431 participants remained in our sample. Our exclusion criteria were determined a priori and were in accordance with our study preregistration.

\section{Procedure}

The procedure of Experiment 2 was similar to Experiment 1, with the exception of the conditions participants were assigned to. Namely, we replaced the Positive Anecdote condition with a Statistics + Positive \& Negative Anecdotes condition to determine whether including a positive anecdote in conjunction with a negative anecdote would lead participants to focus on summary statistics.

We made two other changes in Experiment 2. First, participants in the Statistics condition were explicitly told both the inefficacy and efficacy rates of B-12 injections in treating chronic headaches. We did this to better equate the salience of the inefficacy rate in the Statistics condition to the conditions in which the negative anecdote appeared. Specifically, participants read that "After a two-year trial with 1,000 participants, their study revealed that B-12 injections failed to work for $12.7 \%$ of participants and worked for $87.3 \%$." Second, we changed the Likert scale for our posttest questions regarding the likelihood of trying B-12 injections and giving B-12 to one's child. These were changed to a six-point Likert scale which ranged from $1=$ "Very unlikely" to $6=$ "Very likely".

\section{Predictions}

As in Experiment 1, we predicted that participants in the Statistics + Positive Anecdote condition would tend to have the most positive beliefs towards B-12 injections. We predicted that when participants in the Statistics condition are explicitly presented with the rate of ineffectiveness, this would raise the salience of the inefficacy of B-12 injections. In turn, this may reduce overall endorsement of the efficacy of B-12 injections relative to the Statistics + Positive Anecdote condition. Finally, we sought to examine whether inclusion of the positive anecdote with the negative anecdote in the Statistics + Positive \& Negative Anecdotes condition would cause participants to primarily attend to the statistical information they received. We suspected that the presence of the positive anecdote would not entirely undercut the effect of the negative anecdote on participants' judgments.

\section{Results}

As in Experiment, we fit a multivariate regression model regressing B12 attitudes on Condition (Reference = Statistics condition) and Pretest beliefs. We set priors on intercepts based on posterior estimates from Experiment 1.

Experiment 2 - Priors

$$
\begin{aligned}
& \beta_{\text {Intercept }[1]} \sim \mathcal{N}(-1.38, .5) \\
& \beta_{\text {Intercept }[2]} \sim \mathcal{N}(1.09, .5) \\
& \beta_{\text {Intercept }[3]} \sim \mathcal{N}(2.19, .5) \\
& \beta_{\text {Intercept }[4]} \sim \mathcal{N}(4.59, .5) \\
& \beta_{\text {Pretest } \sim \mathcal{N}(2,4)} \\
& \text { All remaining } \beta \text { were distributed as } \mathcal{N}(0,1) \\
& \Omega_{k} \sim L K J(1) \\
& \text { Group-level parameters were distributed as } \\
& t(3,0,10)
\end{aligned}
$$

These analyses replicated the effects of Experiment 1, showing that (1) the Statistics and Statistics + Positive Anecdote conditions did not differ from each other and (2) that participants in the Statistics + Negative Anecdote condition were more likely to discount the statistical evidence from the clinical trial (see Figure 2), $b_{B 12}=-1.34$, $95 \%$ CI $[-1.89,-0.80] ; b_{\text {Try }}=-0.45,95 \%$ CI $[-1.22,0.28]$; $b_{\text {Child }}=-0.94,95 \%$ CI $[-1.75,-0.19]$. The positive anecdote in the Statistics + Positive and Negative Anecdotes condition, however, did not consistently improve participants' integration of the statistical information, and in some cases, did not differ at all from when participants only received the negative anecdote (see Figure 2), $b_{B 12}=-1.40$, 


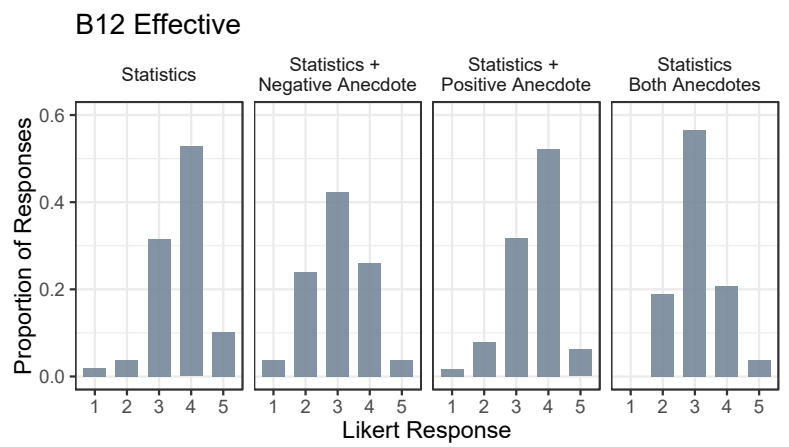

Try B12

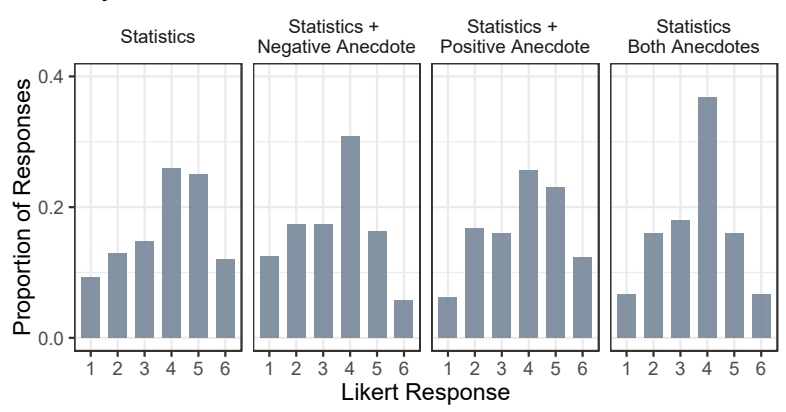

Give B12 to child

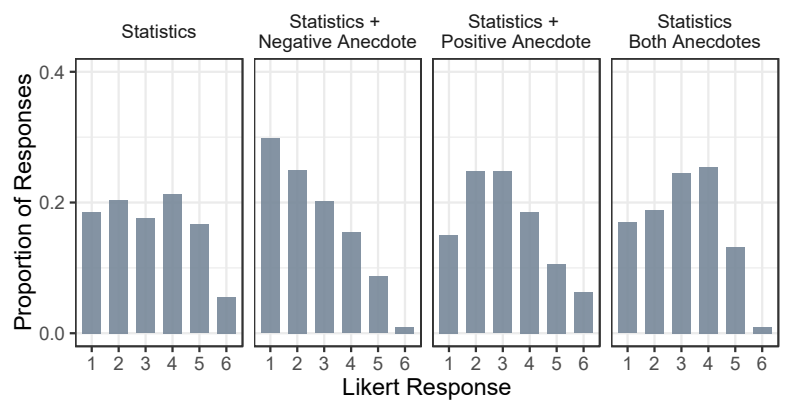

Figure 2: B-12 injection beliefs across conditions in Experiment 2. Higher Likert scale choices indicate more favorable attitudes towards B12 injections. The figure indicates that participants in the Statistics + Negative Anecdote and Statistics + Both Anecdotes conditions had less favorable attitudes towards B12 injections after the intervention relative to participants in the other conditions.

$95 \%$ CI $[-1.93,-0.87] ; b_{T r y}=-0.22,95 \%$ CI [ $\left.-0.96,0.52\right]$; $b_{\text {Child }}=-0.02,95 \%$ CI $[-0.74,0.74]$. These effects again did not interact with people's pretest attitudes towards B12 vaccines.

\section{Experiment 3}

Experiment 2 revealed that presentation of a negative anecdote raises the salience of the inefficacy of B-12 injections. This effect was not consistently negated by positive anecdotal information, raising the question of what means could undercut negative anecdotal information.

Experiment 3 sought to address two questions. First, we addressed the possibility that participants did not realize the anecdote they read was about a person in the study. Our hope was that by visually showing participants that the anecdote they read was about a person in the study we could rule out the possibility that a negative anecdote had its effects just in virtue of it being new, negatively-valenced information. Second, inspired by recent work, Experiment 3 tested whether a visual aid would reduce the impact of the negative anecdote on participants reasoning by making the strength of the summary statistics more salient. Several recent studies suggest that icon arrays, for instance, can improve understanding of scientific consensus (Lewandowsky, Gignac, \& Vaughan, 2013; Nyhan \& Reifler, 2018). Thus, Experiment 3 used an icon array to reduce the effect of the negative anecdote on people's beliefs.

\section{Participants}

We recruited 1,622 participants through Amazon's Mechanical Turk (54\% women, $\left.M_{a g e}=38\right)$. Participants were paid $\$ 0.50$ for participating in the study. After excluding participants who missed questions checking their attention, 1,539 participants remained in our sample. Our exclusion criteria were determined a priori and were in accordance with our study preregistration.

\section{Procedure}

The procedure of Experiment 3 was similar to that of Experiments 1 and 2. Participants were randomly assigned to one of four conditions in a 2 (Icon Array: Present or Absent) $\times 2$ (Negative Anecdote: Present or Absent) between-subjects design. All four conditions included the summary statistical information from the Statistics condition in Experiment 1, allowing us to internally replicate our results in a larger sample.

In the Icon Array only condition, participants first read the statistic about the efficacy of B-12 injections as a medical treatment. They were then shown an icon array showing the success rate of B-12 in 100 people. Participants were then told:

"This image is a depiction of the effectiveness of B-12 as a medical treatment. Imagine 100 people received B-12 injections. The blue figures represent participants that would benefit from the B-12 injections. The green figures represent participants who would fail to benefit from the B-12 injections."

In the Icon Array + Negative Anecdote condition, participants received the same information as the Icon Array only condition but were then told they would read about the experience of one of the subjects in the study and an icon array was displayed with one of the participants circled (see Figure 3), clearly indicating that the anecdote was from someone who participated in the clinical trial.

\section{Predictions}

We predicted that we would replicate the effect of negative anecdotes on participants' acceptance of strong statistical 


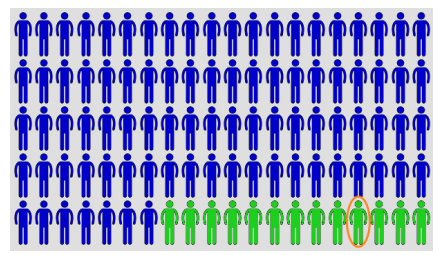

Figure 3: The icon array used in the Icon Array + Negative Anecdote condition in Experiment 3. For the Icon Array only condition, the same array was presented, but without the orange circle.

evidence, as we found in Experiments 1 and 2. We also predicted that in the Icon Array + Negative Anecdote condition, the presence of the icon array would weaken the effect of the negative anecdote (indicating an Icon $x$ Anecdote interaction).

\section{Results}

We fit a Bayesian multivariate regression model regressing B12 attitudes on the interaction between Icon Array $($ Reference $=$ No Array $)$ and Anecdote $($ Reference $=$ No Anecdote), controlling for pretest beliefs:

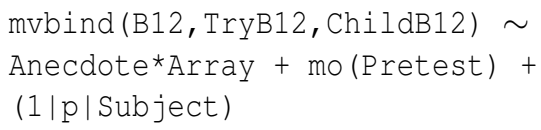

Experiment 3 - Priors

$$
\begin{aligned}
& \beta_{\text {Intercept }[1]} \sim \mathcal{N}(-1.38, .5) \\
& \beta_{\text {Intercept }[2]} \sim \mathcal{N}(1.09, .5) \\
& \beta_{\text {Intercept }[3]} \sim \mathcal{N}(2.19, .5) \\
& \beta_{\text {Intercept }[4]} \sim \mathcal{N}(4.59, .5) \\
& \beta_{\text {Pretest }} \sim \mathcal{N}(4,2)
\end{aligned}
$$$$
\text { All remaining } \beta \text { were distributed as } \mathcal{N}(0,1)
$$$$
\Omega_{k} \sim \operatorname{LKJ}(1)
$$$$
\text { Group-level parameters were distributed as }
$$$$
t(3,0,10)
$$

We replicated the effects of Experiments 1 and 2, showing that a negative anecdote affected participants' integration of statistical information, $b_{B 12}=-1.49,95 \%$ CI $[-1.83,-1.16]$; $b_{\text {Try }}=-1.21,95 \%$ CI $[-1.74,-0.70] ; b_{\text {Child }}=-0.87,95 \% \mathrm{CI}$ $[-1.31,-0.45]$. Consistent with prior work, we also found that providing an icon array improved people's integration of statistical information $\left(b_{B 12}=1.02,95 \%\right.$ CI $[0.69,1.35]$; $b_{\text {Try }}=0.89,95 \%$ CI $[0.39,1.42] ; b_{\text {Child }}=0.86,95 \%$ CI $[0.42,1.30])$, but we observed little evidence for an interaction between these factors, $b_{B 12}=0.00,95 \% \mathrm{CI}$ $[-0.46,0.45] ; b_{T r y}=-0.10,95 \%$ CI $[-0.77,0.58] ; b_{\text {Child }}=$ $-0.33,95 \%$ CI $[-0.89,0.24]$. These results suggest that the negative anecdote nonetheless impacted people's reasoning even when an icon array was present and removed all ambiguity that the anecdote concerned someone who was in the clinical trial.
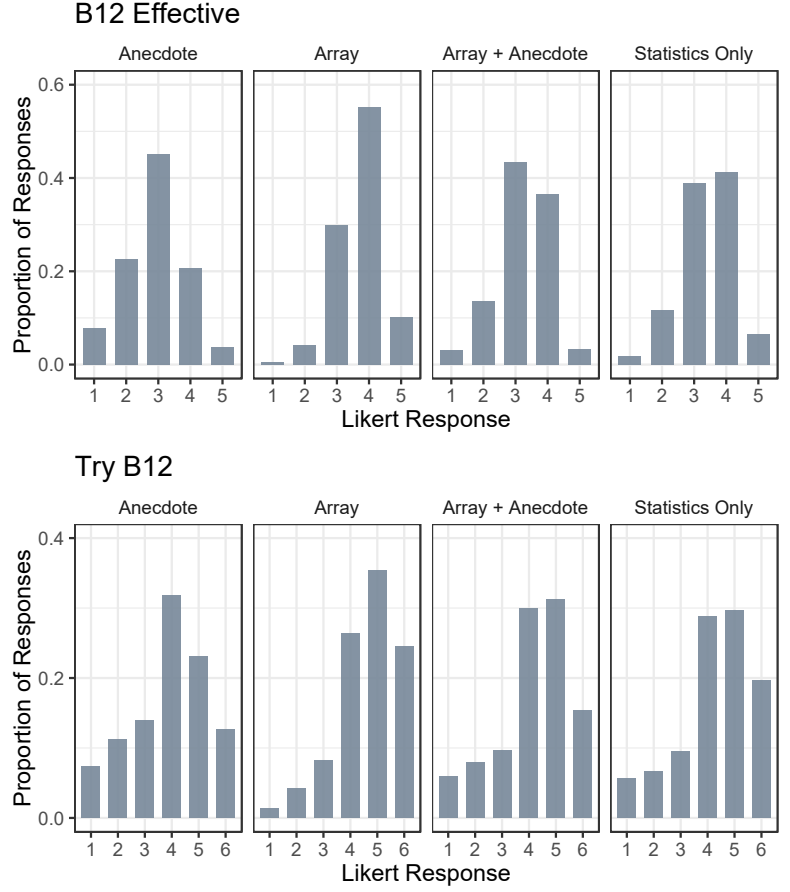

Give B12 to child

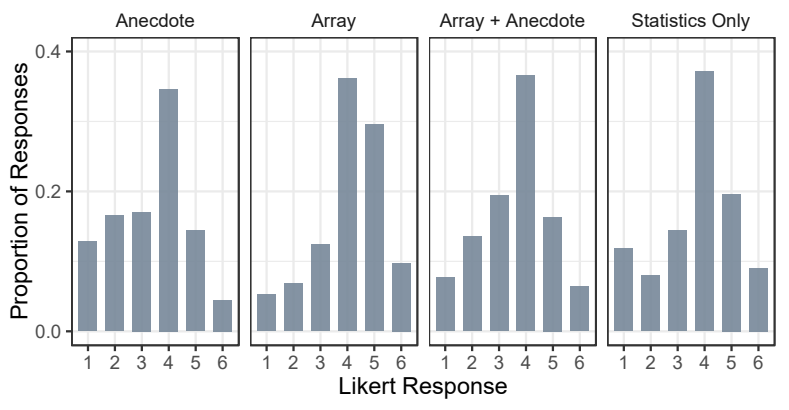

Figure 4: B-12 injection beliefs across conditions in Experiment 3. Higher Likert scale choices indicate more favorable attitudes towards B12 injections. The figure indicates that participants in the Anecdote condition had less favorable attitudes towards B12 injections than participants in the other three conditions.

\section{Discussion}

People have access to more medical information than ever before. From journal articles to online forums, people must determine what information is relevant and reliable to make medical decisions. How do people make these decisions? In three experiments, we tested how people reason about a medical treatment when provided with statistical or anecdotal information. In Experiment 1, we found that a negative anecdote caused people to ignore strong statistical information even though the anecdote involved no negative side effects-indeed, the information presented in the anecdote was already captured by the summary statistics presented to participants. In Experiment 2, we explored whether providing a positive anecdote in addition to a 
negative anecdote would counteract the effect of the negative anecdote. We found that emphasizing a positive outcome of a clinical trial did not consistently undo the effect of the negative anecdote. In Experiment 3, we found that introducing icon arrays improved integration of statistical information overall, but even in this case, anecdotal information negatively impacted people's beliefs. This suggests that a single negative anecdote can carry substantial, unwarranted weight when making a medical decision.

It is striking that a negative anecdote led people to discount strong summary statistics even though the patient was described as suffering no negative side effects because of their treatment. Indeed, we were careful to describe B-12 injections as failing to benefit people who participated in the clinical trial. In reality, many medical treatments involve an element of risk, and some treatments can even involve severe side effects. In these situations, anecdotes that contain new information and highlight side effects would, if anything, yield a larger negative impact on people's ability to properly integrate statistical information. We can see evidence of these effects today: In 2019, vaccine hesitancy was listed as one of the top ten threats to global health (World Health Organization, 2019). In 2018 only $91.1 \%$ (compared to the recommended $95 \%$ ) of children in the United States who were eligible for vaccines received the MMR (measles, mumps, and rubella) vaccine (Centers for Disease Control and Prevention, 2019). 2018 saw the second-highest number of measles cases since 2000. In part, vaccine hesitancy is a consequence of (1) people relying on discredited research linking vaccines to autism and (2) improper reliance on anecdotal information spread in forums purporting to demonstrate the side effects vaccines can wreak on young children (Powell et al., 2018). Our findings can help us make sense of this tendency. Anecdotes carry more weight than they should, as evidenced by the fact that they affected people's reasoning even when they were captured by summary statistics and involved no side effects, highlighting a serious obstacle to public health and demanding new interventions to overcome people's tendency to rely on anecdotal reasoning more than they should.

\section{References}

Allen, M., Bruflat, R., Fucilla, R., Kramer, M., McKellips, S., Ryan, D., \& Spiegelhoff, M. (2000). Testing the persuasiveness of evidence: Combining narrative and statistical evidence. Communication Research Reports, 17(4), 331-336.

Allen, M., \& Preiss, R. (1997). Comparing the persuasiveness of narrative and statistical evidence. Communication Research Reports, 14(2), 125-131.

Brody, J., Annett, R., Scherer, D., Perryman, M., \& Cofrin, K. (2005). Comparisons of adolescent and parent willingness to participate in minimal and above-minimal risk pediatric asthma research protocols. Journal of Adolescent Health, 37(3), 229-235.

Bürkner, P., \& Charpentier, E. (2018). Monotonic effects: A principled approach for including ordinal predictors in regression models. PsyArXiv.

Bürkner, P. C. (2018). Advanced Bayesian multilevel modeling with the $\mathrm{R}$ package brms. The R Journal, 10(1), 395-411.

Centers for Disease Control and Prevention. (2019). Measles cases and outbreaks. Retrieved from https://www.cdc.gov/measles/cases-outbreaks.html.

Dahlstrom, M. (2014). Using narratives and storytelling to communicate science with nonexpert audiences. Proceedings of the National Academy of Sciences, 111(4), 13614-13620.

Hornikx, J. (2005). A review of experimental research on the relative persuasiveness of anecdotal, statistical, causal, and expert evidence. Studies in Communication Sciences, 5(1), 205-216.

Hornikx, J. (2018). Combining anecdotal and statistical evidence in real-life discourse: Comprehension and persuasion. Discourse Processes, 55(3), 324-336.

Johnson, F., Özdemir, S., Mansfield, C., Hass, S., Siegel, C., $\&$ Sands, B. (2009). Are adult patients more tolerant of treatment risks than parents of juvenile patients? Risk Analysis, 29(1), 121-136.

Lewandowsky, S., Gignac, G. E., \& Vaughan, S. (2013). The pivotal role of perceived scientific consensus in acceptance of science. Nature Climate Change, 3(4), 399 - 404.

Liddell, T., \& Kruschke, J. (2018). Analyzing ordinal data with metric models: What could possibly go wrong? Journal of Experimental Social Psychology, 79, 328-348.

McElreath, R. (2016). Statistical rethinking: A bayesian course with examples in $R$ and Stan. Boca Raton, FL: CRC Press.

Nyhan, B., \& Reifler, J. (2018). The roles of information deficits and identity threat in the prevalence of misperceptions. Journal of Elections, Public Opinions and Parties, 1 - 23.

Powell, D., Weisman, K., \& Markman, E. (2018). Articulating lay theories through graphical models: A study of beliefs surrounding vaccination decisions. Proceedings of the 40th Annual Conference of the Cognitive Science Society.

Rodriguez, F., Rhodes, R., Miller, K., \& Shah, P. (2016). Examining the influence of anecdotal stories and the interplay of individual differences on reasoning. Thinking \& Reasoning, 22(3), 274-296.

Shen, F., Sheer, V., \& Li, R. (2015). Impact of narratives on persuasion in health communication: A meta-analysis. Journal of Advertising, 44(2), 105-113.

World Health Organization. (2019). Ten threats to global health in 2019. Retrieved from https://www.who.int/emergencies/ten-threats-to-globalshealth-in-2019. 